PROCEEDINGS OF THE

AMERICAN MATHEMATICAL SOCIETY

Volume 125, Number 3, March 1997, Pages 907-915

S 0002-9939(97)03683-6

\title{
POSITIVE SCALAR CURVATURE AND ODD ORDER ABELIAN FUNDAMENTAL GROUPS
}

\author{
REINHARD SCHULTZ
}

(Communicated by Thomas Goodwillie)

\begin{abstract}
If a smooth manifold has a Riemannian metric with positive scalar curvature, it follows immediately that the universal covering also has such a metric. The paper establishes a converse if the manifold in question is closed of dimension at least 5 and the fundamental group is an elementary abelian $p$-group of rank 2 , where $p$ is an odd prime.
\end{abstract}

A conjecture of J. Rosenberg [Rs3] states that a closed smooth manifold with odd order fundamental group and dimension $\geq 5$ admits a Riemannian metric with positive scalar curvature if and only if its universal covering admits such a metric. Standard considerations involving transfers ( $c f$. [KS1]) reduce the conjecture to the special case of $p$-groups (where $p$ is an odd prime). Results of Rosenberg [Rs1][Rs3] and S. Kwasik and the author [KS1] prove the conjecture if the fundamental group $G$ is a cyclic $p$-group. In this work we study the conjecture when $G$ is a finite abelian $p$-group. The following interim conclusion reflects many of the basic ideas and disposes of the first examples not covered by [KS1], [Rs1]-[Rs3].

Theorem. Let $p$ be an odd prime, and let $M^{n}$ be a closed smooth manifold of dimension $n \geq 5$ with fundamental group $\mathbb{Z}_{p} \times \mathbb{Z}_{p}$. Then $M$ admits a Riemannian metric with positive scalar curvature if and only if its universal covering $\widetilde{M}$ does.

In [RsS] J. Rosenberg and S. Stolz prove a stable result that yields a weaker conclusion for $G \cong \mathbb{Z}_{p} \times \mathbb{Z}_{p}$ but applies to all finite groups. In particular, if $G$ is a finite group of odd order and $M$ is a closed smooth manifold with fundamental group $G$, their result states that the universal covering $\widetilde{M}$ has a Riemannian metric with positive scalar curvature if and only if some product $M \times X \times \cdots \times X$ does, where $X$ is an 8-dimensional Bott manifold; i.e., it is a spin manifold whose $\widehat{A}$-genus is equal to 1 . Although the methods should yield quantitative information on the number of factors of $X$ that are needed, it is not clear how precise such estimates would be. Our result implies that if $G \approx \mathbb{Z}_{p} \times \mathbb{Z}_{p}$, then no copies of $X$ are needed if $n \geq 5$ and at most one copy of $X$ is needed if $n<5$. A more geometric proof of this result in dimensions $\leq 2 p-2$ was obtained independently by Stolz (unpublished).

It seems likely that the methods of this paper can be combined with the multiple Künneth formula decomposition of $[\mathrm{Hn}]$ and finite induction to prove at least a semistable version of Rosenberg's conjecture for all elementary abelian $p$-groups

Received by the editors February 13, 1995 and, in revised form, September 13, 1995.

1991 Mathematics Subject Classification. Primary 53C21, 55N15, 57R75; Secondary 53C20, $57 \mathrm{R} 85$.

(C) 1997 American Mathematical Society 
(where $p$ is odd). We hope to study this problem in a subsequent paper. On the other hand, the computations become substantially more complicated even if one considers a fundamental group of the form $\mathbb{Z}_{p^{2}} \times \mathbb{Z}_{p}$ (as usual, $p$ is assumed to be odd).

\section{Preliminary observations}

The proof of the theorem is a combination of formal considerations along the lines of [RsS] and [KS1] and a detailed analysis of the real connective $K$-theory of $B\left(\mathbb{Z}_{p} \times \mathbb{Z}_{p}\right)$. We shall begin with the formal considerations.

Reduction to questions involving connective $K$-theory. As in [Rs1]-[Rs3] and [KS1], it suffices to show that all classes in

$$
\begin{gathered}
\widetilde{\Omega}_{*}^{\text {Spin }}\left(B\left(\mathbb{Z}_{p} \times \mathbb{Z}_{p}\right)\right)=\operatorname{Kernel}\left(\Omega_{*}^{\text {Spin }}\left(B\left(\mathbb{Z}_{p} \times \mathbb{Z}_{p}\right)\right) \rightarrow \Omega_{*}^{\text {Spin }}(\mathrm{pt} .)\right) \\
(\cong \text { corresponding kernel with } S O \text { replacing Spin })
\end{gathered}
$$

of dimension $\geq 5$ have representatives that support Riemannian metrics with positive scalar curvature. In other words, if $\operatorname{Pos}_{n}\left(B\left(\mathbb{Z}_{p} \times \mathbb{Z}_{p}\right)\right)$ is the subset of $\Omega_{n}^{\text {Spin }}\left(B\left(\mathbb{Z}_{p} \times \mathbb{Z}_{p}\right)\right)$ with representatives of this type, then it suffices to show that $\operatorname{Pos}_{n}\left(B\left(\mathbb{Z}_{p} \times \mathbb{Z}_{p}\right)\right)$ contains $\widetilde{\Omega}_{n}^{\text {Spin }}\left(B\left(\mathbb{Z}_{p} \times \mathbb{Z}_{p}\right)\right)$; since $\operatorname{Pos}_{n}\left(B\left(\mathbb{Z}_{p} \times \mathbb{Z}_{p}\right)\right)$ is in fact a subgroup, the use of algebraic methods in this connection should seem promising. Furthermore, if $D: \Omega_{*}^{\text {Spin }} \rightarrow b o_{*}$ is the transformation from Spin bordism to connective real $K$-theory given by the Dirac operator (equivalently by the Clifford orientation of a spin bundle), then results of R. Jung $[\mathrm{J}]$ and the standard algebraic formalism for bordism theories (e.g., [B] and [L1]) show that $\operatorname{Pos}_{n}\left(B\left(\mathbb{Z}_{p} \times \mathbb{Z}_{p}\right)\right)$ contains the kernel of $D_{*}$. Therefore, if we define $\operatorname{Pos}_{n}^{k o}\left(B\left(\mathbb{Z}_{p} \times \mathbb{Z}_{p}\right)\right)$ to be the image of $\operatorname{Pos}_{n}\left(B\left(\mathbb{Z}_{p} \times \mathbb{Z}_{p}\right)\right)$ under $D_{*}$, then it suffices to show that $\operatorname{Pos}_{n}^{k o}\left(B\left(\mathbb{Z}_{p} \times \mathbb{Z}_{p}\right)\right)$ contains the reduced homology group $\widetilde{b o_{n}}\left(B\left(\mathbb{Z}_{p} \times \mathbb{Z}_{p}\right)\right)$.

Stable splittings of classifying spaces. A result of R. Holzsager [Ho] states that the suspension $\Sigma B \mathbb{Z}_{p}$ splits into a wedge of $p-1$ summands, say $Y_{1} \vee \cdots \vee Y_{p-1}$, such that

$$
\widetilde{H}_{s}\left(Y_{j}, \mathbb{Z}\right) \cong \begin{cases}\mathbb{Z}_{p} & \text { if } s \equiv 2 j \bmod 2(p-1), \\ 0 & \text { otherwise. }\end{cases}
$$

Since $B\left(\mathbb{Z}_{p} \times \mathbb{Z}_{p}\right)$ is homotopy equivalent to $B \mathbb{Z}_{p} \times B \mathbb{Z}_{p}$, it follows that $\Sigma^{2}\left(B\left(\mathbb{Z}_{p} \times \mathbb{Z}_{p}\right)\right)$ is homotopy equivalent to a wedge of two copies of $\Sigma^{2} B \mathbb{Z}_{p}$ and one copy of $\Sigma B \mathbb{Z}_{p} \wedge \Sigma B \mathbb{Z}_{p}$. Holzsager's result yields a corresponding stable splitting of $B\left(\mathbb{Z}_{p} \times \mathbb{Z}_{p}\right)$ (i.e., in a suitable category of spectra) into a wedge of $p^{2}-1$ summands

$$
\left(\bigvee_{r=1}^{p-1} X_{r, 0}\right) \vee\left(\bigvee_{s=1}^{p-1} X_{0, s}\right) \vee\left(\bigvee_{u, v=1}^{p-1} X_{u, v}\right)
$$

where $X_{r, 0}$ and $X_{0, s}$ are equivalent to $\Sigma^{-1} Y_{r}$ and $\Sigma^{-1} Y_{s}$ respectively, and $X_{u, v}$ is equivalent to $\Sigma^{-2} Y_{u} \wedge Y_{v}$. This splitting is a refinement of the decomposition in [MP].

Splittings of positively curved classes in connective $K$-theory. The splitting of the previous paragraph and formal properties of generalized homology theories yield an associated splitting of $\Omega_{*}^{S O}\left(B\left(\mathbb{Z}_{p} \times \mathbb{Z}_{p}\right)\right)$, and results from [KS1, Prop. 1.7, p. 276] yield a corresponding splitting of $\operatorname{Pos}_{n}^{k o}\left(B\left(\mathbb{Z}_{p} \times \mathbb{Z}_{p}\right)\right)$; in other words, if $\alpha$ lies in the latter group and $\alpha=\prod \alpha_{j}$ is the direct sum decomposition, then each $\alpha_{j}$ also 
lies in $\operatorname{Pos}_{n}^{k o}\left(B\left(\mathbb{Z}_{p} \times \mathbb{Z}_{p}\right)\right)$. More generally, if $G$ is an arbitrary finite group and we are given a stable splitting of $B G$ as a wedge $W_{1} \vee W_{2}$, then $\operatorname{Pos}_{n}^{k o}\left(W_{i}\right)$ will denote the projection of $\operatorname{Pos}_{n}^{k o}(B G)$ into $b o_{n}\left(W_{i}\right)$ for $i=1,2$. In this notation the splitting principle of this paragraph can be restated as the formula

$$
\operatorname{Pos}_{n}^{k o}(B G) \approx \operatorname{Pos}_{n}^{k o}\left(W_{1}\right) \oplus \operatorname{Pos}_{n}^{k o}\left(W_{2}\right) \text {. }
$$

\section{Computations}

Our analysis of $b o_{*}\left(B\left(\mathbb{Z}_{p} \times \mathbb{Z}_{p}\right)\right)$ is based upon the Atiyah-Hirzebruch spectral sequence

$$
H_{s}\left(B\left(\mathbb{Z}_{p} \times \mathbb{Z}_{p}\right) ; b o_{t}(\{\text { pt. }\})\right) \Rightarrow b o_{s+t}\left(B\left(\mathbb{Z}_{p} \times \mathbb{Z}_{p}\right)\right)
$$

We shall denote the terms of this spectral sequence by $E_{s, t}^{r}\left(B\left(\mathbb{Z}_{p} \times \mathbb{Z}_{p}\right)\right)$. The stable splitting of $B\left(\mathbb{Z}_{p} \times \mathbb{Z}_{p}\right)$ in the previous section yields an algebraic splitting of this spectral sequence into the Atiyah-Hirzebruch spectral sequences for the spectra $X_{u, v}$ for $0 \leq u, v \leq p-1$ (with the convention that $X_{0,0}$ is a point). We shall denote these summands by $E_{s, t}^{r}\left(X_{u, v}\right)$. Similarly, if we are given an arbitrary stable splitting of $B G$ (where $G$ is a finite group) as a wedge $W_{1} \vee W_{2}$, then we obtain a splitting of $E_{s, t}^{r}(B G)$ into two spectral sequences that we shall call $E_{s, t}^{r}\left(W_{1}\right)$ and $E_{s, t}^{r}\left(W_{2}\right)$.

We shall first prove the main theorem for even dimensional manifolds; the argument in these cases is extremely simple.

Proposition 1. The conclusion of the theorem holds if $n$ is even.

Proof. Since $p$ is odd the $E^{2}$ terms of the Atiyah-Hirzebruch spectral sequence $\left\{E_{s, t}^{r}\left(B\left(\mathbb{Z}_{p} \times \mathbb{Z}_{p}\right)\right)\right\}$ vanish for $s>0$ unless $t \equiv 0 \bmod 4$, and if $t=4 t^{\prime}$ (where $\left.t^{\prime} \geq 0\right)$ then $E_{s, t}^{2}$ is isomorphic to $H_{s}\left(\mathbb{Z}_{p} \times \mathbb{Z}_{p} ; \mathbb{Z}\right)$. Since the integral homology of $\mathbb{Z}_{p}$ has one copy of $\mathbb{Z}_{p}$ in each odd positive dimension and nothing in even positive dimensions, the Künneth formula implies that

$$
H_{s}\left(\mathbb{Z}_{p} \times \mathbb{Z}_{p} ; \mathbb{Z}\right) \cong \bigoplus_{i} H_{i}\left(\mathbb{Z}_{p} ; \mathbb{Z}\right) \otimes H_{s-i}\left(\mathbb{Z}_{p} ; \mathbb{Z}\right)
$$

provided $s$ is even.

If we combine this with the multiplicative properties of the Atiyah-Hirzebruch spectral sequence and collapsing of the corresponding spectral sequence

$$
H_{s}\left(B \mathbb{Z}_{p} ; b o_{t}(\{\mathrm{pt.}\})\right) \Rightarrow b o_{s+t}\left(B \mathbb{Z}_{p}\right)
$$

we see that all classes in $E_{s, t}^{2}\left(B\left(\mathbb{Z}_{p} \times \mathbb{Z}_{p}\right)\right)$ survive to $E^{\infty}$ if $s+t$ is even. The multiplicativity of the projection from Spin bordism to connective real $K$-theory implies that the product map in the latter is compatible with the obvious geometric product map in Spin bordism. Since $\operatorname{Pos}_{n}^{k o}\left(B\left(\mathbb{Z}_{p}\right)\right)=\widetilde{b o_{n}}\left(\mathbb{Z}_{p}\right)$ if $n \geq 3$ by [Rs1] and the product of two manifolds with metric of positive scalar curvature also has such a metric, these observations combine to show that every class in $b o_{n}\left(B\left(\mathbb{Z}_{p} \times \mathbb{Z}_{p}\right)\right)$ lies in $\operatorname{Pos}_{n}^{k o}\left(B\left(\mathbb{Z}_{p} \times \mathbb{Z}_{p}\right)\right)$ if $n$ is even.

Corollary 2. If $s+t$ is odd, then the differentials into $E_{s, t}^{r}\left(B\left(\mathbb{Z}_{p} \times \mathbb{Z}_{p}\right)\right)$ are all zero and $E_{s, t}^{\infty}\left(B\left(\mathbb{Z}_{p} \times \mathbb{Z}_{p}\right)\right)$ is (isomorphic to) a subgroup of $E_{s, t}^{2}\left(B\left(\mathbb{Z}_{p} \times \mathbb{Z}_{p}\right)\right)$.

This follows immediately because all terms in even degrees are permanent cycles. 
Remarks. (1) It is well known that the Atiyah-Hirzebruch spectral sequence for the unitary bordism homology groups $\Omega_{*}^{U}\left(B\left(\mathbb{Z}_{p} \times \mathbb{Z}_{p}\right)\right)$ does not collapse (e.g., see [L2]). By Proposition 1 the nontriviality of this spectral sequence implies the existence of nontrivial differentials from terms of odd total degree to terms of even total degree in the Atiyah-Hirzebruch spectral sequence for $b o_{*}\left(B\left(\mathbb{Z}_{p} \times \mathbb{Z}_{p}\right)\right)$.

(2) In contrast to the preceding result, the Atiyah-Hirzebruch spectral sequences

$$
H_{s}\left(B \mathbb{Z}_{p} ; h_{t}\left(B \mathbb{Z}_{p}\right)\right) \Rightarrow h_{s+t}\left(B\left(\mathbb{Z}_{p} \times \mathbb{Z}_{p}\right)\right)
$$

associated to the usual product fibration of the space $B \mathbb{Z}_{p} \times B \mathbb{Z}_{p} \approx B\left(\mathbb{Z}_{p} \times \mathbb{Z}_{p}\right)$ do collapse for $h_{*}=\Omega_{*}^{U}$ or $b o_{*}$ (cf. [Rw, Prop. 6, p. 888] for the case $h_{*}=\Omega_{*}^{S O}$ ).

The odd dimensional case. We shall first introduce some more notation. If $h_{*}$ is a connective homology theory, $m$ is a nonnegative integer and $X$ is a $C W$ complex or connective spectrum, then $\mathcal{F}^{m}\left(h_{n}(X)\right)$ will denote the subgroup of elements in $h_{n}(X)$ that have filtration $\leq m$ in the Atiyah-Hirzebruch spectral sequence

$$
H_{s}\left(X ; h_{t}(\{\mathrm{pt} .\})\right) \Rightarrow h_{s+t}(X) .
$$

If $G$ is a finite group, then $\operatorname{Pos}_{*}^{k o} E_{s, t}^{\infty}(B G)$ will denote the subquotient of $\operatorname{Pos}_{s+t}^{k o}(B G)$ given by

$$
\begin{array}{r}
\mathcal{F}^{s-1}\left(b o_{s+t}(B G)\right)+\operatorname{Pos}_{s+t}^{k o}(B G) \cap \mathcal{F}^{s}\left(b o_{s+t}(B G)\right) / \mathcal{F}^{s-1}\left(b o_{s+t}(B G)\right) \\
\approx \operatorname{Pos}_{s+t}^{k o}(B G) \cap \mathcal{F}^{s}\left(b o_{s+t}(B G)\right) / \operatorname{Pos}_{s+t}^{k o}(B G) \cap \mathcal{F}^{s-1}\left(b o_{s+t}(B G)\right)
\end{array}
$$

and if we are given a stable splitting of $B G$ as a wedge $W_{1} \vee W_{2}$, then $\operatorname{Pos}_{*}^{k o} E_{s, t}^{\infty}\left(W_{1}\right)$ and $\operatorname{Pos}_{*}^{k o} E_{s, t}^{\infty}\left(W_{2}\right)$ are defined similarly (no parentheses are needed in the expression $\mathcal{F}^{s-1}+\operatorname{Pos}_{*}^{k o} \cap \mathcal{F}^{s}$ because the lattice of subgroups of an abelian group is modular).

The following result provides the basic lower estimate for $\operatorname{Pos}_{*}^{k o} E_{s, t}^{\infty}\left(B\left(\mathbb{Z}_{p} \times \mathbb{Z}_{p}\right)\right)$ when $s$, or equivalently $s+t$, is odd; the equivalence of the latter two conditions follows because $E_{s, t}^{2}=0$ unless $t \equiv 0 \bmod 4$.

Proposition 3. In the setting above assume that $s$ is odd, $0 \leq t \equiv 0 \bmod 4$, and $s+t \geq 3$. Then $\operatorname{Pos}_{*}^{k o} E_{s, t}^{\infty}\left(B\left(\mathbb{Z}_{p} \times \mathbb{Z}_{p}\right)\right)$ is a $\mathbb{Z}_{p}$ vector space that contains a subspace of dimension $\frac{s+3}{2}$ or $p+1$ depending on whether $s \leq 2 p-1$ or $s \geq 2 p+1$.

Proof. The vector space assertion follows because the Künneth formula implies that $E_{s, t}^{2}\left(B\left(\mathbb{Z}_{p} \times \mathbb{Z}_{p}\right)\right)$ is a $\mathbb{Z}_{p}$ vector space if $s>0$.

If $i_{1}, i_{2}: \mathbb{Z}_{p} \rightarrow \mathbb{Z}_{p}$ are the injections onto the first and second summands, then naturality properties of $[\mathrm{KS} 1]$ imply that $\operatorname{Pos}_{n}^{k o}\left(B\left(\mathbb{Z}_{p} \times \mathbb{Z}_{p}\right)\right)$ contains $i_{1 *} \operatorname{Pos}_{n}^{k o}\left(B \mathbb{Z}_{p}\right)$ and $i_{2 *} \operatorname{Pos}_{n}^{k o}\left(B \mathbb{Z}_{p}\right)$. Since $\operatorname{Pos}_{n}^{k o}\left(B \mathbb{Z}_{p}\right)=\widetilde{b o_{n}}\left(B \mathbb{Z}_{p}\right)$ by [Rs1] it follows that

$$
\operatorname{Pos}_{n}^{k o}\left(B\left(\mathbb{Z}_{p} \times \mathbb{Z}_{p}\right)\right) \supset i_{1 *} \widetilde{b o_{n}}\left(B \mathbb{Z}_{p}\right)+i_{2 *} \widetilde{b o_{n}}\left(B \mathbb{Z}_{p}\right) \text {. }
$$

By the Künneth formula we have

$$
\mathcal{F}^{1}\left(b o_{n}\left(B\left(\mathbb{Z}_{p} \times \mathbb{Z}_{p}\right)\right)\right)=i_{1 *} \mathcal{F}^{1}\left(b o_{n}\left(B \mathbb{Z}_{p}\right)\right)+i_{2 *} \mathcal{F}^{1}\left(b o_{n}\left(B \mathbb{Z}_{p}\right)\right),
$$

and if $s=1$ the proposition follows from this and the results of [Rs1].

Now assume $s>1$ is odd, and write $s=2 m-1$ for some $m \geq 2$. Let $L^{2 m-1}$ be the standard lens space, and consider the element $\Delta_{m}$ in $b o_{2 m-1}\left(B\left(\mathbb{Z}_{p} \times \mathbb{Z}_{p}\right)\right)$ arising from the bordism class of the map $L^{2 m-1} \rightarrow B \mathbb{Z}_{p} \rightarrow B\left(\mathbb{Z}_{p} \times \mathbb{Z}_{p}\right)$ where $L \rightarrow B \mathbb{Z}_{p}$ is 2-connected and $d_{\#}: B \mathbb{Z}_{p} \rightarrow B\left(\mathbb{Z}_{p} \times \mathbb{Z}_{p}\right)$ is induced by the diagonal map $d: \mathbb{Z}_{p} \rightarrow$ $\mathbb{Z}_{p} \times \mathbb{Z}_{p}$. Since the lens space has a Riemannian metric with constant positive 
sectional curvature, it follows immediately that $\Delta_{m}$ lies in $\operatorname{Pos}_{2 m-1}^{k o}\left(B\left(\mathbb{Z}_{p} \times \mathbb{Z}_{p}\right)\right)$. If $U: b o_{*} \rightarrow H_{*}(-; \mathbb{Z})$ is the transformation that detects the generator of $b o_{0}(\{\mathrm{pt}\}$.$) ,$ then $U\left(\Delta_{m}\right)$ is nontrivial and therefore $\Delta_{m}$ defines a nontrivial element of

$$
E_{s, 0}^{\infty}\left(B\left(\mathbb{Z}_{p} \times \mathbb{Z}_{p}\right)\right) \subset E_{s, 0}^{2}\left(B\left(\mathbb{Z}_{p} \times \mathbb{Z}_{p}\right)\right) \approx H_{s}\left(\mathbb{Z}_{p} \times \mathbb{Z}_{p} ; \mathbb{Z}\right)
$$

(where $s=2 m-1$ as before). Specifically, if we consider the Künneth formula splitting

$$
\begin{aligned}
& H_{2 m-1}\left(\mathbb{Z}_{p} \times \mathbb{Z}_{p} ; \mathbb{Z}\right) \cong\left(H_{2 m-1}\left(\mathbb{Z}_{p}\right) \otimes \mathbb{Z}\right) \oplus\left(\mathbb{Z} \otimes H_{2 m-1}\left(\mathbb{Z}_{p}\right)\right) \\
& \oplus \bigoplus_{i+j=m} \operatorname{Tor}\left(H_{2 i-1}\left(\mathbb{Z}_{p} ; \mathbb{Z}\right), H_{2 j-1}\left(\mathbb{Z}_{p} ; \mathbb{Z}\right)\right),
\end{aligned}
$$

then the cup product structure of $H^{*}\left(L ; \mathbb{Z}_{p}\right)$ implies that the projection of $\Delta_{m}$ onto each of these summands is nontrivial.

The next step in the proof is to show that the projections of $\Delta_{m}$ into the groups $H_{s}\left(X_{u, v} ; \mathbb{Z}\right)$ span a $\mathbb{Z}_{p}$ vector space whose dimension is given as in the statement of the proposition. This is an immediate consequence of the last sentence in the previous paragraph and the following elementary observation:

Proposition 4. With respect to the Künneth formula decomposition above and the stable splitting of $B\left(\mathbb{Z}_{p} \times \mathbb{Z}_{p}\right)$, all nontrivial maps from the Künneth formula summands to the stable homotopy summands are given as follows:

(1) $H_{2 m-1}\left(\mathbb{Z}_{p}\right) \otimes \mathbb{Z}$ maps nontrivially to $H_{2 m-1}\left(X_{M, 0}\right)$, where $m \equiv M \bmod p-1$.

(2) $\mathbb{Z} \otimes H_{2 m-1}\left(\mathbb{Z}_{p}\right)$ maps nontrivially to $H_{2 m-1}\left(X_{0, M}\right)$, where $m \equiv M \bmod p-1$.

(3) $\operatorname{Tor}\left(H_{2 i-1}\left(\mathbb{Z}_{p} ; \mathbb{Z}\right), H_{2 j-1}\left(\mathbb{Z}_{p} ; \mathbb{Z}\right)\right)$ maps nontrivially to $H_{2 m-1}\left(X_{I, J}\right)$, where $i \equiv I \bmod p-1$ and $j \equiv J \bmod p-1$.

The proof is elementary and left to the reader.

If $s \leq 2 p-1$, then Proposition 4 implies that the individual Künneth formula summands belong to distinct stable summands of $B\left(\mathbb{Z}_{p} \times \mathbb{Z}_{p}\right)$, and this means that

$$
\operatorname{dim} \operatorname{Pos}_{*}^{k o} E_{s, 0}^{\infty}\left(B\left(\mathbb{Z}_{p} \times \mathbb{Z}_{p}\right)\right) \geq \frac{s+3}{2}=\operatorname{dim} H_{s}\left(\mathbb{Z}_{p} \times \mathbb{Z}_{p} ; \mathbb{Z}\right)
$$

The reverse inequality also holds because $\operatorname{Pos}_{*}^{k o} E_{s, 0}^{\infty}\left(B\left(\mathbb{Z}_{p} \times \mathbb{Z}_{p}\right)\right)$ is a subgroup of $E_{s, 0}^{2}\left(B\left(\mathbb{Z}_{p} \times \mathbb{Z}_{p}\right)\right)$ and the latter is isomorphic to $\operatorname{dim} H_{s}\left(\mathbb{Z}_{p} \times \mathbb{Z}_{p} ; \mathbb{Z}\right)$. On the other hand, if $s \geq 2 p+1$, then it follows that the projections of $\Delta_{m}$ onto the $p+1$ groups $\operatorname{Pos}_{*}^{k o} E_{s, 0}^{\infty}\left(X_{0, M}\right), \operatorname{Pos}_{*}^{k o} E_{s, 0}^{\infty}\left(X_{M, 0}\right)$, and $\operatorname{Pos}_{*}^{k o} E_{s, 0}^{\infty}\left(X_{M, 0}\right)$ are linearly independent, where $M$ is defined as in the statement of Proposition 4 and for each $i$ satisfying $1 \leq i \leq p-1$ the integer $l(i)$ is uniquely specified by the conditions $1 \leq l(i) \leq p-1$ and $i+l(i) \equiv m \bmod p-1$. If we take $W_{s, 0}$ to be (i) all of $E_{s, 0}^{2}$ if $s \leq 2 p-1$ and $W_{s, 0}$, (ii) the span of the $p+1$ projections of $\Delta_{m}$ if $s \geq 2 p+1$, then the preceding discussion proves Proposition 3 when $t=0$.

We shall prove the corresponding result for $t>0$ using the periodicity properties of connective real $K$-theory. First, recall that $b o_{*}$ has a multiplicative structure arising from the tensor product of vector bundles and this structure is well behaved with respect to the Atiyah-Hirzebruch spectral sequence. Next, recall that $E_{s, t}^{2}\left(B\left(\mathbb{Z}_{p} \times \mathbb{Z}_{p}\right)\right) \neq 0$ for $s>0$ only if $t=4 l$ for some $l>0$. In fact, if $\mathbf{v}$ generates $b o_{4}(\{$ pt. $\})$, then multiplication by $\mathbf{v}$ induces isomorphism $E_{s, t}^{2} \approx E_{s, t+4}^{2}$ for all $s>0$ and $t \geq 0$. Also, if $s$ is odd, then multiplication by $\mathbf{v}$ sends $E_{s, t}^{\infty}$ into $E_{s, t+4}^{\infty}$. 
Combining the observations of this paragraph with the previous work we see that

$$
\mathbf{v}^{l} \operatorname{Pos}_{*}^{k o} E_{s, 0}^{\infty} \subset E_{s, 4 l}^{\infty}
$$

for all $s, l>0$. Since (i) multiplication by $\mathbf{v}$ is realized geometrically by taking products with the Kummer surface, (ii) the set of manifolds with metrics of positive scalar curvature is closed under taking products with arbitrary manifolds, it follows that $\mathbf{v}^{l} \operatorname{Pos}_{*}^{k o} E_{s, 0}^{\infty}$ is in fact contained in $\operatorname{Pos}_{*}^{k o} E_{2,4 l}^{\infty}$. Furthermore, since multiplication by $\mathbf{v}$ is injective on $E^{2}$ and all differentials in the Atiyah-Hirzebruch spectral sequence for $b o_{*}\left(B\left(\mathbb{Z}_{p} \times \mathbb{Z}_{p}\right)\right)$ go from odd total degrees to even total degrees (by Corollary 2), it also follows that multiplication by $\mathbf{v}$ is injective on $E^{\infty}$. Therefore the conclusion of Proposition 3 follows in these cases by taking $W_{s, 4 l}=\mathbf{v}^{l} W_{s, 0}$.

Corollary 5 (independently due to Stolz). In the setting of Proposition 3 we have $\operatorname{Pos}_{*}^{k o} E_{s, t}^{\infty}=E_{s, t}^{\infty}=E_{s, t}^{2}$ for $s \leq 2 p-1$.

The following refinement of Proposition 3 is useful for our purposes.

Proposition 6. In the setting of Proposition 3 if $s$ and $n$ are odd such that $n \geq$ $s \geq 2 p-1$, then

$$
\mathcal{W}_{n, s, 2 p-1}=\mathcal{F}^{s}\left(b o_{n}\left(B\left(\mathbb{Z}_{p} \times \mathbb{Z}_{p}\right)\right)\right) / \mathcal{F}^{s-2 p-2}\left(b o_{n}\left(B\left(\mathbb{Z}_{p} \times \mathbb{Z}_{p}\right)\right)\right)
$$

is $a \mathbb{Z}_{p}$ vector space, and it contains a subspace of dimension $\geq \frac{p^{2}-1}{2}$ for which every element lifts to a class in $\operatorname{Pos}_{n}^{k o}\left(B\left(\mathbb{Z}_{p} \times \mathbb{Z}_{p}\right)\right)$.

Sketch of proof. By construction the nonzero integral homology of the stable summand $X_{u, v}$ of $B\left(\mathbb{Z}_{p} \times \mathbb{Z}_{p}\right)$ only appears in dimensions congruent to $2(u+v)-1$ or $2(u+v)-2 \bmod 2 p-2$. In particular, this means that the entire spectral sequence splits into $p-1$ pieces such that $E_{s, t}^{r}$ of the $i$ th spectral sequence is trivial unless $s \equiv 2 i-\varepsilon \bmod 2 p-2$, where $\varepsilon=1$ or 2 . In particular, this implies that $\mathcal{F}^{s} / \mathcal{F}^{s-2 p+2}$ is isomorphic to a direct sum

$$
\bigoplus_{i=0}^{p-1} \mathcal{F}^{s-i} / \mathcal{F}^{s-i-1}
$$

(these filtrations all refer to $b o_{n}\left(B\left(\mathbb{Z}_{p} \times \mathbb{Z}_{p}\right)\right)$; we have suppressed this term for the sake of conciseness). The proposition follows by combining the observations in the preceding sentences with Proposition 3.

The next order of business is to find an upper bound for the quotient appearing in the statement of Proposition 6:

Proposition 7. The dimension of the $\mathbb{Z}_{p}$ vector space

$$
\mathcal{W}_{n, s, 2 p-1}=\mathcal{F}^{s}\left(b o_{n}\left(B\left(\mathbb{Z}_{p} \times \mathbb{Z}_{p}\right)\right)\right) / \mathcal{F}^{s-2 p-2}\left(b o_{n}\left(B\left(\mathbb{Z}_{p} \times \mathbb{Z}_{p}\right)\right)\right)
$$

is at most $\frac{p^{2}-1}{2}$.

Since every subgroup of an abelian group with $\leq q$ generators is also generated by $\leq q$ elements, Proposition 7 will be an immediate consequence of the following more general statement.

Proposition 8. If $n$ is odd, then $\widetilde{b o_{n}}\left(B\left(\mathbb{Z}_{p} \times \mathbb{Z}_{p}\right)\right)$ is generated by at most $\frac{p^{2}-1}{2}$ elements. 
Notational convention. If $h_{*}$ is a generalized homology theory we shall denote its localization away from 2 by $h_{*}\left[\frac{1}{2}\right]$.

Proof. Consider first the analogous question for $\widetilde{b o_{n}}\left(B \mathbb{Z}_{p}\right)$ where $n$ is odd. We claim that the latter is generated by at most $\frac{p-1}{2}$ elements. There are many proofs of this; for the sake of completeness here is an elementary argument: Let $L^{N}$ be a lens space of dimension $N>n$; then it suffices to show that the torsion subgroup of $\widetilde{b o_{n}}\left(L^{N}\right)$ is generated by at most $\frac{p-1}{2}$ elements. Since the Atiyah-Hirzebruch spectral sequence for connective real $K$-theory and the periodic localized theory $K O_{*}\left[\frac{1}{2}\right]$ both collapse, it follows that the composite

$$
\widetilde{b o_{n}}\left(L^{N}\right) \rightarrow K O_{n}\left(L^{N}\right) \rightarrow K O_{n}\left[\frac{1}{2}\right]\left(L^{N}\right)
$$

is injective, and thus it suffices to verify the statement about generators for the torsion subgroup of $\widetilde{K O_{n}}\left[\frac{1}{2}\right]\left(L^{N}\right)$. Since $K O_{*}\left[\frac{1}{2}\right]$ is a direct summand of $K_{*}\left[\frac{1}{2}\right]$ it follows that every weakly almost complex manifold is orientable with respect to $K O_{*}\left[\frac{1}{2}\right]$, and therefore $K O_{n}\left[\frac{1}{2}\right]\left(L^{N}\right)$ is isomorphic to $K O^{N-n}\left[\frac{1}{2}\right]\left(L^{N}\right)$ by Poincaré duality. Since $N$ and $n$ are both odd and $\widetilde{b o_{n}}\left(L^{N}\right)$ is odd torsion, it suffices to know that the torsion in $\widetilde{K O^{N-n}}\left(L^{N}\right)$ is generated by at most $\frac{p-1}{2}$ elements. This follows immediately from the standard description of the complex $K$-theory of lens spaces ( $c f .\left[\right.$ A, p. 103]), the identification of $K O\left[\frac{1}{2}\right]$ with the self-conjugate elements of $K\left[\frac{1}{2}\right]$, and Bott periodicity.

We shall derive Proposition 8 by combining the ideas of the preceding paragraph with the Künneth formula for complex $K$-theory [A, Cor. 2.7.15, pp. 113-114] and the standard identification of $K O_{*}\left[\frac{1}{2}\right]$ as the self-conjugate part of $K_{*}\left[\frac{1}{2}\right]$ (i.e., the direct summand inclusion mentioned above). Clearly it suffices to prove the proposition for the product $L \times L$ instead of $B\left(\mathbb{Z}_{p} \times \mathbb{Z}_{p}\right)$ because the canonical map $L \times L \rightarrow B\left(\mathbb{Z}_{p} \times \mathbb{Z}_{p}\right)$ is $N$-connected. The same reasoning as in the proof of Proposition 1 shows that all differentials in the Atiyah-Hirzebruch spectral sequences for $\widetilde{b o}_{*}\left[\frac{1}{2}\right](L \times L)$ and $\widetilde{K O_{*}}\left[\frac{1}{2}\right](L \times L)$ are trivial in terms of even total degree, and therefore it follows that the canonical map from $\widetilde{b o_{*}}\left[\frac{1}{2}\right](L \times L)$ to $\widetilde{K O_{*}}\left[\frac{1}{2}\right](L \times L)$ is injective. This in turn reduces the proof of Proposition 8 to verifying the upper bound for the torsion subgroup of $\widetilde{K O_{*}}\left[\frac{1}{2}\right](L \times L)$.

Since $L \times L$ is orientable with respect to complex $K$-theory (and hence also $\left.K O_{*}\left[\frac{1}{2}\right]\right)$, it follows by Poincaré duality that $\widetilde{K O_{n}}\left[\frac{1}{2}\right](L \times L)$ is isomorphic to

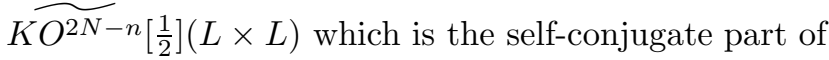

$$
\widetilde{K^{2 N-n}}\left[\frac{1}{2}\right](L \times L) \approx \widetilde{K^{1}}\left[\frac{1}{2}\right](L \times L)
$$

because $n$ is odd. By the Künneth formula for complex $K$-theory and Poincaré duality the torsion subgroup of the latter is isomorphic to

$$
\left(\widetilde{K^{0}}\left[\frac{1}{2}\right](L) \otimes \widetilde{K^{1}}\left[\frac{1}{2}\right](L)\right) \oplus\left(\widetilde{K^{1}}\left[\frac{1}{2}\right](L) \otimes \widetilde{K^{0}}\left[\frac{1}{2}\right](L)\right) \oplus \operatorname{Tor}\left(\widetilde{K^{0}}\left[\frac{1}{2}\right](L), \widetilde{K^{0}}\left[\frac{1}{2}\right](L)\right)
$$

with the usual restricted naturality properties. Now $K^{1}(L)=\mathbb{Z}$ and conjugation acts as \pm 1 depending upon the class of $N$ mod 4 . Therefore each of the first two summands is given by either the self-conjugate or skew elements of $\widetilde{K^{0}}\left[\frac{1}{2}\right](L) \approx$ $\widetilde{K^{0}}(L)$ depending upon $N \bmod 4$. It is well known that each of these summands is 
generated by at most $\frac{p-1}{2}$ elements. On the other hand, the self-conjugate part of the Tor term is the direct sum

$$
\operatorname{Tor}\left(\widetilde{K_{+}^{0}}\left[\frac{1}{2}\right](L), \widetilde{K_{+}^{0}}\left[\frac{1}{2}\right](L)\right) \oplus \operatorname{Tor}\left(\widetilde{K_{-}^{0}}\left[\frac{1}{2}\right](L), \widetilde{K_{-}^{0}}\left[\frac{1}{2}\right](L)\right)
$$

where $K_{ \pm}\left[\frac{1}{2}\right]$ denotes the self-conjugate or skew portion of $K\left[\frac{1}{2}\right]$ depending upon the subscript. If $A$ and $B$ are finite abelian groups, it is elementary to check that the minimum number of generators for $\operatorname{Tor}(A, B)$ is at most the product of the minimum numbers of generators for $A$ and $B$ respectively, and therefore it follows that each summand is generated by at most $\frac{(p-1)^{2}}{4}$ elements. If we combine all these upper estimates with the Künneth formula decomposition we see that the torsion subgroups in question have generating sets with at most $\frac{p^{2}-1}{2}$ elements.

\section{Proof of the MAIN THEOREM}

By Proposition 1 the theorem is true if $n$ is even, so it suffices to consider the case where $n \geq 5$ is odd. We shall prove by induction on $s$ that $\operatorname{Pos}_{n}^{k o}\left(B\left(\mathbb{Z}_{p} \times \mathbb{Z}_{p}\right)\right)$ contains $\mathcal{F}^{s}\left(\widetilde{b o_{n}}\left(B\left(\mathbb{Z}_{p} \times \mathbb{Z}_{p}\right)\right)\right)$ for all $s$ satisfying $1 \leq s \leq n$. If $s=1$ this follows from Corollary 5 and the identity

$$
\mathcal{F}^{1}\left(\widetilde{b o}_{n}\left(B\left(\mathbb{Z}_{p} \times \mathbb{Z}_{p}\right)\right)\right)=E_{1, n-1}^{\infty}\left(B\left(\mathbb{Z}_{p} \times \mathbb{Z}_{p}\right)\right) .
$$

Assume that the result holds for $s-1$ where $s \geq 2$; if $\mathcal{F}^{s-1}=\mathcal{F}^{s}$, then of course there is nothing to prove, so it suffices to consider the cases where the filtration inclusion is proper. Since this only happens if $s$ is odd, we shall assume this henceforth.

Suppose first that $s \leq 2 p-1$. In this case Corollary 5 implies that

$$
\mathcal{F}^{s}\left(\widetilde{b o_{n}}\left(B\left(\mathbb{Z}_{p} \times \mathbb{Z}_{p}\right)\right)\right) \subset \operatorname{Pos}_{n}^{k o}\left(B\left(\mathbb{Z}_{p} \times \mathbb{Z}_{p}\right)\right)+\mathcal{F}^{s-1}\left(\widetilde{b o_{n}}\left(B\left(\mathbb{Z}_{p} \times \mathbb{Z}_{p}\right)\right)\right),
$$

and since $\operatorname{Pos}_{n}^{k o}\left(B\left(\mathbb{Z}_{p} \times \mathbb{Z}_{p}\right)\right)$ contains $\mathcal{F}^{s-1}\left(\widetilde{b o_{n}}\left(B\left(\mathbb{Z}_{p} \times \mathbb{Z}_{p}\right)\right)\right)$ by induction, it follows that the former also contains $\mathcal{F}^{s}$ (same).

Suppose next that $s \geq 2 p+1$. Let $\mathcal{W}_{n, s, 2 p-2}$ be the subquotient appearing in the statements of Propositions 6 and 7, and let $\mathcal{W}_{n, s, 2 p-2}^{+}$be the subspace

$$
\mathcal{F}^{s-2 p+2}\left(b o_{n}(B G)\right)+\operatorname{Pos}_{n}^{k o}(B G) \cap \mathcal{F}^{s}\left(b o_{n}(B G)\right) / \mathcal{F}^{s-2 p+2}\left(b o_{n}(B G)\right)
$$

where we write $G=\mathbb{Z}_{p} \times \mathbb{Z}_{p}$ in order to save space. Proposition 6 implies that $\mathcal{W}_{n, s, 2 p-2}$ is a $\mathbb{Z}_{p}$ vector space, and by Proposition 7 its dimension is at most $\frac{p^{2}-1}{2}$. On the other hand, Proposition 6 also implies that $\operatorname{dim} \mathcal{W}_{n, s, 2 p-2}^{+} \geq \frac{p^{2}-1}{2}$, and therefore we must have $\mathcal{W}_{n, s, 2 p-2}^{+}=\mathcal{W}_{n, s, 2 p-2}$. By the definitions of these vector spaces it follows that

$$
\left.\mathcal{F}^{s}\left(\widetilde{b o_{n}}\left(B\left(\mathbb{Z}_{p} \times \mathbb{Z}_{p}\right)\right)\right) \subset \mathcal{F}^{s-2 p+2} \widetilde{\left(b o_{n}\right.}\left(B\left(\mathbb{Z}_{p} \times \mathbb{Z}_{p}\right)\right)\right)+\operatorname{Pos}_{n}^{k o}\left(B\left(\mathbb{Z}_{p} \times \mathbb{Z}_{p}\right)\right) .
$$

Since $\left.\mathcal{F}^{s-2 p+2} \widetilde{\left(b o_{n}\right.}\left(B\left(\mathbb{Z}_{p} \times \mathbb{Z}_{p}\right)\right)\right)$ is contained in $\operatorname{Pos}_{n}^{k o}\left(B\left(\mathbb{Z}_{p} \times \mathbb{Z}_{p}\right)\right)$ by induction, we therefore have $\mathcal{F}^{s}\left(\widetilde{b o_{n}}\left(B\left(\mathbb{Z}_{p} \times \mathbb{Z}_{p}\right)\right)\right) \subset \operatorname{Pos}_{n}^{k o}\left(B\left(\mathbb{Z}_{p} \times \mathbb{Z}_{p}\right)\right)$ as required.

\section{ACKNowledgments}

This paper is an outgrowth of earlier joint work with Sławomir Kwasik [KS1, KS2]. I am grateful to him for many suggestions and insights regarding this subject. I am also grateful to Jonathan Rosenberg and Stephan Stolz for providing preprints and verbal accounts of their work, and to the Max-Planck-Institut für Mathematik 
and Tulane University for their hospitality and support during portions of the work on this paper.

\section{REFERENCES}

[A] M. Atiyah, K-theory (notes by D. W. Anderson), Addison-Wesley, Reading, Mass., 1989. MR 90m: 18011

[B] N. Baas, On the bordism theory of manifolds with singularities, Math. Scand. 33 (1973), 279-302. MR 49:11547b

[BeS] J. C. Becker and R. E. Schultz, Equivariant function spaces and stable homotopy theory, Comment. Math. Helv. 49 (1974), 1-34. MR 49:3994

[Ho] R. Holzsager, Stable splitting of $K(G, 1)$, Proc. Amer. Math. Soc. 31 (1972), 305-306. MR 44:4744

[Hn] T. Hungerford, Multiple Künneth formulas for abelian groups, Trans. Amer. Math. Soc. 118 (1965), 257-275. MR 31:229

[J] R. Jung, Ph. D. Thesis, Univ. Mainz, in preparation.

[KS1] S. Kwasik and R. Schultz, Positive scalar curvature and periodic fundamental groups, Comment. Math. Helv. 65 (1990), 271-286. MR 91k:57027

[KS2] _ Fake spherical spaceforms with constant positive scalar curvature, Comment. Math. Helv. 71 (1996), 1-40. CMP 96:07

[L1] P. Landweber, Künneth formulas for bordism theories, Trans. Amer. Math. Soc. 121 (1966), 242-256. MR 33:728

[L2] - Complex bordism of classifying spaces, Proc. Amer. Math. Soc. 27 (1971), 175-179. MR 42:3782

[MP] S. Mitchell and S. Priddy, Stable splittings derived from the Steinberg module, Topology 22 (1983), 285-298. MR 85f:55005

[Rs1] J. Rosenberg, $C^{*}$-algebras, positive scalar curvature, and the Novikov Conjecture, Publ. Math. I. H. E. S. 58 (1983), 197-212. MR 85g:58083

[Rs2] $\longrightarrow C^{*}$-algebras, positive scalar curvature, and the Novikov Conjecture-II, Geometric Methods in Operator Algebras (Kyoto, 1983), Pitman Res. Notes Math. Ser. 123, Longman Sci. Tech., Harlow, U. K., pp. 341-374. MR 88f:58140

[Rs3],$C^{*}$-algebras, positive scalar curvature, and the Novikov Conjecture-III, Topology 25 (1986), 319-336. MR 88f:58141

[RsS] J. Rosenberg and S. Stolz, A "stable" version of the Gromov-Lawson conjecture, Contemp. Math., vol. 181, Amer. Math. Soc., Providence, RI, 1995, pp. 405-418. CMP 95:09

[Rw] R. Rowlett, Free actions of a p-group on two generators, Indiana Univ. Math. J. 26 (1977), 885-889. MR 56:16656

Department of Mathematics, Purdue University, West Lafayette, Indiana 47907

Current address: Department of Mathematics, University of California, Riverside, California 92521

E-mail address: schultz@math.ucr.edu 